\title{
Multipolynomial Resultants and Linear Algebra
}

\author{
Dinesh Manocha and John F. Canny \\ Computer Science Division \\ University of California \\ Berkeley, CA 94720 \\ USA
}

\begin{abstract}
The problem of eliminating variables from a set of polynomial equations arises in many symbolic and numeric applications. The three main approaches are resultants, Gröbner bases and the Wu-Ritt method. In practice, resultant based algorithms have been shown to be most effectice for certain applications. The resultant of a set of polynomial equations can be expressed in terms of matrices and determinants and the bottleneck in its computation is the symbolic expansion of determinants. In this paper we present interpolation based algorithms to compute symbolic determinants. The main characteristic of the algorithms is the use of techniques from linear algebra to reduce the symbolic complexity of the computation and number of function evaluations. These include linearizing a matrix polynomial to its companion form and similarity transformations for reduction to upper Hessenberg form followed by reduction to Frobenius canonical form. We consider dense as well as sparse interpolation algorithms. These algorithms have been implemented as part of a package for resultant computation and we discuss their performance for certain applications.
\end{abstract}

\section{Introduction}

Computational methods to manipulate sets of polynomial equations are gaining importance in symbolic and numeric computation. The fundamental problems include simultaneous elimination of one or more variables to obtain a "symbolically smaller" system and computing the numeric solutions of a system of equations. Elimination theory, a branch of classical algebraic geometry, presents constructive approaches

Permission to copy without fee all or part of this material is granted provided that the copies are not made or distributed for direct commercial advantage, the ACM copyright notice and the title of the publication and its date appear, and notice is given that copying is by permission of the Association for Computing Machinery. To copy otherwise, or to republish, requires a fee and/or specific permission.

ISSAC' $92-7 / 92 / C A$, USA

- 1992 ACM 0-89791-490-2/92/0007/0158...\$1.50 for these problems. The main result is the construction of a single resultant polynomial of $n$ homogeneous equations in $n$ unknowns, such that its vanishing is an exact condition for the given system of equations to have a non-trivial solution [Mac64, Sal85, Wae50]. The resultant is a polynomial in the coefficients of the equations. We refer this resultant as the multipolynomial resultant of the given system of equations [MC91b].

The process of eliminating variables from a system of linear equations is widely known. Most of the theory expresses the conditions in terms of matrices and determinants. Elimination theory deals with generalizing these techniques to system of nonlinear equations and reducing the problem to solving system of linear equations. The most familiar form of the resultant is the Sylvester's formulation for two nonlinear equations, which expresses it as determinant of a matrix. However, a single determinant formulation may not exist for all systems of nonlinear equations. The most general formulation, to the best of our knowledge, expresses the resultant as a ratio of two determinants as formulated by Macaulay [Mac02, Mac21]. Many a times both the determinants evaluate to zero and the resultant is being computed by perturbation and limiting arguments [Can88]. This corresponds to the ratio of the characteristic polynomials of two matrices and is termed as the Generalized Characteristic polynomial as introduced by Canny [Can90].

There are two other algorithmic approaches known in the literature for eliminating variables from a system of equations. The first one is based on polynomial ideal theory and generates special bases for polynomial ideals, called Gröbner bases. The algorithm for Gröbner bases is due to Buchberger and is surveyed in [Buc85, Buc89]. Eliminating a set of variables is a special application of Gröbner bases. The second approach for variable elimination has been developed by Wu Wen-Tsün [Wu78] using an idea 
proposed by Ritt [Rit50]. This approach is based on Ritt's characteristic set construction and successfully applied to automated geometry theorem proving by Wu. It is referred as the Ritt-Wu's algorithm [GC90]. All these methods of elimination are surveyed in [KL92]. When it comes to practice resultants have been shown to be more effective for applications like implicitization [MC91a]. Moreover, the resulting algorithms are fast in practice and it is possible to obtain a tight bound on their running time. On the other hand Gröbner bases and characteristic sets computations may be slow for even small problems. As a result it is difficult to derive tight bounds on the running time of the computation.

The algorithm for resultant computation involves the use of suitable resultant formulation and reduces the problem to expanding symbolic determinants. The entries of the matrices are polynomial functions in terms of the coefficients of the given polynomial equations. Manocha and Canny use multivariate interpolation and modular techniques for computing symbolic determinants [MC91a]. Multivariate interpolation converts the computation into integer number crunching as opposed to generating and manipulating intermediate symbolic expressions. In particular, the algorithm involves numeric determinant computations for function evaluation and using sparse or dense multivariate interpolation for resultant polynomial computation. It turns out that function evaluation is relatively expensive and for many applications accounts for almost half of the running time. Moreover, Macaulay's formulation generates two matrices whose order is much greater than the degree of the resultant [Mac02, Mac21]. As a result, a great deal of time is spent in numeric determinant evaluations and thereby slowing down the interpolation algorithm.

In this paper, we present improved algorithms for computing symbolic determinants using multivariate polynomial interpolation. We use a variety of techniques from linear algebra to decrease the number of function evaluations and the symbolic complexity of the resulting computation. These include linearizing a matrix polynomial to an equivalent blcok companion matrix followed by similarity transformations for reduction to upper Hessenberg form and finally to Frobenius canonical form. These transformations are used along with sparse and dense interpolation algorithms and as a result the interpolation problem is divided into smaller problems of lower complexity. They also lead to improved and faster algorithms for solving system of non linear equations based on $\mathrm{u}$-resultants as highlighted in [Can88, MC91b] and computing the generalized characteristic polynomi- als for a system of equations [Can90].

The rest of the paper is organized in the following manner. In Section 2 we present a brief review of multipolynomial resultants and linear algebra. In Section 3 we present a simple and efficient algorithm for computing univariate symbolic determinant, whose entries are polynomials in one variable. This algorithm is based on techniques from linear algebra as opposed to interpolation. The algorithm is extended to multivariate symbolic determinants using dense and sparse algorithms for multivariate interpolation in Section 4. We describe its implementation and performance for implicitization Section 5 . In particular, we obtain considerable speed-ups as compared to earlier algorithms.

\section{Background}

Multipolynomial resultants are the oldest and by now the best known methodology for eliminating variables. Most of their development began in the last century. Given a system of $n+1$ affine algebraic equations in $n+m$ unknowns $x_{1}, \ldots, x_{n}, y_{1}, \ldots, y_{m}$, the resultant is a polynomial in $y_{1}, \ldots, y_{m}$. In this paper, we assume that the coefficients of the polynomials are rational numbers, though the results are extendible to any field of characteristic zero. In case, the coefficients are represented as floating point numbers, we treat them as rational numbers and use exact arithmetic for the rest of the computation. Geometrically, the zero set of the resultant represents the projection of the algebraic set defined by the intersection of the zero sets of the $n+1$ equations. In case $m=0$, the vanishing of the resultant becomes a necessary and sufficient condition for the given system of equations to have a non-trivial solution. There are many specialized formulations of resultants for lower values of $n$ (up to six), which express it as the determinant of a matrix, as opposed to the general formulation expressing it as a ratio of two determinants [Dix08, Jou89, MC27, Sal85, Stu91, SZ91].

In the rest of the paper we use upper case bold face letters to denote matrices and lower case bold face letters to represent 1-dimensional vectors. All the other symbols represent scalar variables.

\subsection{Linear Algebra}

Algorithms for resultant computation deal with matrices and determinants. As a result, we utilize many properties from linear algebra for efficient resultant computation. In this section we review some techniques from linear algebra and use them 
in the following sections. More details are given in [GLR82, Wil65].

Matrix Polynomials If $\mathbf{A}_{0}, \mathbf{A}_{1}, \ldots, \mathbf{A}_{k}$ are $m \times m$ numeric matrices on rationals, then the matrixvalued function defined on the rationals by $\mathbf{L}(\lambda)=$ $\sum_{i=0}^{k} \mathbf{A}_{i} \lambda^{i}$ is called a matrix polynomial of degree $\mathrm{k}$. When $\mathbf{A}_{k}=\mathbf{I}$, the identity matrix, the matrix polynomial is said to be monic.

Let us consider the case when $\mathbf{A}_{k}$ is a nonsingular matrix. Let

$\bar{L}(\lambda)=\mathbf{A}_{k}^{-1} \mathbf{L}(\lambda)$, and $\overline{\mathbf{A}}_{i}=\mathbf{A}_{k}^{-1} \mathbf{A}_{8}, \quad 0 \leq i<k$.

$\bar{L}(\lambda)$ is a monic matrix polynomial. According to Theorem 1.1 [GLR82], the determinant of $\bar{L}(\lambda)$ corresponds exactly to the characteristic polynomial of

$$
\mathbf{C}=\left[\begin{array}{ccccc}
\mathbf{0} & \mathbf{I}_{m} & \mathbf{0} & \ldots & \mathbf{0} \\
\mathbf{0} & \mathbf{0} & \mathbf{I}_{m} & \ldots & \mathbf{0} \\
\vdots & \vdots & \ldots & \vdots & \vdots \\
\mathbf{0} & \mathbf{0} & \mathbf{0} & \ldots & \mathbf{\mathbf { I }}_{m} \\
-\overline{\mathbf{A}}_{0} & -\overline{\mathbf{A}}_{1} & -\overline{\mathbf{A}}_{2} & \ldots & -\overline{\mathbf{A}}_{k-1}
\end{array}\right]
$$

where $\mathbf{0}$ and $\mathbf{I}_{m}$ are $m \times m$ null and identity matrices, respectively. We refer $\mathbf{C}$ as the block companion matrix associated with the matrix polynomial $\overline{\mathbf{L}}(\lambda)$. In other words, the eigenvalues of $\mathbf{C}$ correspond exactly to the roots of Determinant $(\mathbf{L}(\lambda))=0$ or Determinant $(\overline{\mathbf{L}}(\lambda))=0$

In the case where $\mathbf{A}_{k}$ is a singular matrix, the matrix polynomial $\mathbf{L}(\lambda)$ is linearized into a companion polynomial $\mathbf{C}_{L}(\lambda)$ of the form [GLR82]:

$$
\begin{gathered}
\mathbf{C}_{L}(\lambda)=\left[\begin{array}{ccccc}
\mathbf{I}_{m} & \mathbf{0} & \mathbf{0} & \ldots & \mathbf{0} \\
\mathbf{0} & \mathbf{I}_{m} & \mathbf{0} & \ldots & \mathbf{0} \\
\vdots & \vdots & \ldots & \vdots & \vdots \\
\mathbf{0} & \mathbf{0} & \ldots & \mathbf{I}_{m} & \mathbf{0} \\
\mathbf{0} & \mathbf{0} & \ldots & \mathbf{0} & \mathbf{A}_{k}
\end{array}\right] \lambda+ \\
{\left[\begin{array}{ccccc}
\mathbf{0} & -\mathbf{I}_{m} & \mathbf{0} & \ldots & \mathbf{0} \\
\mathbf{0} & \mathbf{0} & -\mathbf{I}_{m} & \ldots & \mathbf{0} \\
\vdots & \vdots & \ldots & \vdots & \vdots \\
\mathbf{0} & \mathbf{0} & \ldots & \mathbf{0} & -\mathbf{I}_{m} \\
\mathbf{A}_{0} & \mathbf{A}_{1} & \ldots & \mathbf{A}_{k-2} & \mathbf{A}_{k-1}
\end{array}\right]}
\end{gathered}
$$

We will represent this linearization as $\mathbf{P} \lambda+\mathbf{Q}$. The determinant of $\mathbf{C}_{L}(\lambda)=\mathbf{P} \lambda+\mathbf{Q}$ corresponds exactly to the determinant of $\mathbf{L}(\lambda)$.

The roots of the characteristic polynomial of a matrix correspond to its eigenvalues. Moreover, the eigenvalues of a matrix $\mathbf{M}$ are invariant under similarity transformations of the form $\overline{\mathbf{M}}=\mathbf{H}^{-1} \mathbf{M H}$, where $\mathbf{H}$ is a non-singular matrix. As a result the characteristic polynomial of the matrix obtained after applying similarity transformations to $\mathbf{C}$ in (1) corresponds exactly to the determinant of $\overline{\mathbf{L}}(\lambda)$.
Upper Hessenberg Matrix

An upper Hessenberg matrix is of the form

$$
\mathbf{S}=\left[\begin{array}{ccccc}
s_{1,1} & s_{1,2} & \ldots & s_{1, m-1} & s_{1, m} \\
s_{2,1} & s_{2,2} & \ldots & s_{2, m-1} & s_{2, m} \\
0 & s_{3,2} & \ldots & s_{3, m-1} & s_{3, m} \\
0 & 0 & \ldots & \ldots & s_{4, m} \\
\vdots & \vdots & \ldots & \vdots & \vdots \\
0 & 0 & \ldots & s_{m, m-1} & s_{m, m}
\end{array}\right]
$$

An upper Hessenberg matrix is reduced, if any of the subdiagonal elements, $s_{i, i-1}$, is zero.

Frobenius Canonical Form Let the characteristic polynomial of $\mathbf{M}$ be

$(-1)^{m}\left[\lambda^{m}-c_{m-1} \lambda^{m-1}-c_{m-2} \lambda^{m-2}-\ldots-c_{0}\right]=0$.

Then the characteristic polynomial of the matrix

$$
\mathbf{C}=\left[\begin{array}{ccccc}
c_{m-1} & c_{m-2} & \ldots & c_{1} & c_{0} \\
1 & 0 & \ldots & 0 & 0 \\
0 & 1 & \ldots & 0 & 0 \\
\vdots & \vdots & \ldots & \vdots & \vdots \\
0 & 0 & \ldots & 1 & 0
\end{array}\right]
$$

is identical to the characteristic polynomial of $\mathbf{M}$. The matrix $\mathbf{C}$ is the companion matrix of the characteristic polynomial of $\mathbf{M}$. A matrix may sometimes be similar to the companion matrix of its characteristic polynomial. The exact criterion for similarity is defined in terms of the multiplicity of the eigenvalues of $\mathbf{M}$ and the structure of its Jordan canonical form [Wil65]. A matrix is called derogatory if it is not similar to the companion matrix of its characteristic polynomial.

A Frobenius matrix $\mathbf{C}_{r}$ of order $r$ is a matrix of the form

$$
\mathbf{C}_{r}=\left[\begin{array}{ccccc}
c_{r-1} & c_{r-2} & \ldots & c_{1} & c_{0} \\
1 & 0 & \ldots & 0 & 0 \\
0 & 1 & \ldots & 0 & 0 \\
\vdots & \vdots & \ldots & \vdots & \vdots \\
0 & 0 & \ldots & 1 & 0
\end{array}\right]
$$

Every matrix $\mathbf{M}$ may be reduced by a similarity transformation to the direct sum of a number $s$ of Frobenius matrices denoted as $\mathbf{C}_{r_{1}}, \mathbf{C}_{r_{2}}, \ldots, \mathbf{C}_{r_{s}}$. The characteristic polynomial of each $\mathbf{C}_{r_{2}}$ divides the characteristic polynomial of all the preceding $\mathbf{C}_{r_{i}}$. In the case of a non-derogatory matrix, $s=1$ and $r_{1}=n$. The direct sum of the Frobenius matrices is called the Frobenius canonical form. The trans- 
formed matrix is represented as

$$
\mathbf{F}=\left[\begin{array}{ccccc}
\mathbf{C}_{r_{1}} & \mathbf{0} & \ldots & \mathbf{0} & \mathbf{0} \\
\mathbf{0} & \mathbf{C}_{r_{2}} & \ldots & \mathbf{0} & \mathbf{0} \\
\vdots & \vdots & \ldots & \vdots & \vdots \\
\mathbf{0} & \mathbf{0} & \ldots & \mathbf{C}_{r_{s-1}} & \mathbf{0} \\
\mathbf{0} & \mathbf{0} & \ldots & \mathbf{0} & \mathbf{C}_{r_{s}}
\end{array}\right]
$$

where $\mathbf{0}$ denotes a null matrix of appropriate order.

\subsection{Univariate Determinants}

In this section we consider the problem of expanding a univariate symbolic determinant. Each entry of the $m \times m$ matrix, $\mathbf{L}(\lambda)$, is a polynomial of degree $k$ in $\lambda$ and let the determinant be a polynomial of degree $d$. The degree $d$ is bounded by $n=k m$. A simple algorithm is evaluating the numeric determilnant for $\lambda=p^{i}$ for $0 \leq i \leq d$, where $p$ is a prime number. This is followed by Vandermonde interpolation to compute the symbolic determinant. Howevel, the running cost of the method is $O\left(d m^{3}+d^{2}\right)$ finite field operations, although the cost of interpolation can be improved by using Kaltofen and Laksman's algorithm [KL88]. In many applications of resultants, each entry of the matrix is a linear polynomial in the coefficients of the nonlinear equations and therefore, $d \leq m$. As a result, the running time of the algorithm is $O\left(d^{4}\right)$ finite field operations. In this section we use techniques from linear algebra and present a simple and improved algorithm of $O\left(d^{3}+n^{3}\right)$ complexity for most cases in practice. This algorithm is repeatedly used in the multivariate interpolation algorithms presented in the next section. We refer to the algorithm as univariate determinant algorithm. The algorithm is:

1. Express $\mathbf{L}(\lambda)=\sum_{i=0}^{k} \mathbf{A}_{i} \lambda^{i}$ as a matrix polynomial.

2. Linearize the matrix polynomial to an equivalent companion matrix $\mathbf{C}$ of order $d \times d$ using the reduction presented in (1).

3. Use similarity transformations to construct an upper Hessenberg matrix, S, similar to $\mathbf{C}$.

4. Transform $\mathbf{S}$ to its Frobenius canonical form using similarity transformations.

5. The characteristic polynomial of $\mathbf{C}, P(\lambda)$, is computed by the product of the of the characteristic polynomials of Frobenius matrices constituting the Frobenius canonical form.

6. Multiply $P(\lambda)$ by the determinant of the leading matrix of $\mathbf{L}(\lambda)$.
The linearization of a matrix polynomial into a companion follows from (1), in case the leading matrix $\mathbf{A}_{k}$ is non-singular. Lets consider the case when the leading matrix is singular.

We use the reduction, (2.1), to linearize the matrix polynomial into a companion matrix of the form $\mathbf{P} \lambda+\mathbf{Q}$. This matrix can be transformed into an equivalent companion matrix using row operations, similar to the ones used in Gaussian elimination.

Let the rows of $\mathbf{P}$ and $\mathbf{Q}$ be represented as $\mathbf{p}_{\boldsymbol{i}}$ and $q_{j}$, respectively. Each matrix is of order $n$. Moreover, their elements are denoted as $p_{i j}$ and $q_{i j}$. We perform elementary row operations on $\mathbf{P}$ to reduce it to an upper triangular form. Each elementary row operation is of the form:

$$
\mathbf{p}_{k}=\mathbf{p}_{k}-\frac{p_{k i}}{p_{i i}} \mathbf{p}_{i}
$$

At each step perform a corresponding operation is performed on $\mathbf{Q}$.

$$
\mathbf{b}_{k}=\mathbf{b}_{k}-\frac{p_{k i}}{p_{i i}} \mathbf{b}_{i}
$$

As a result the determinant of $\mathbf{P} \lambda+\mathbf{Q}$ remains a constant, where $\mathbf{P}$ and $\mathbf{Q}$ are updated with row operations (like Gaussian elimination).

After the row operations $\mathbf{P}$ and $\mathbf{Q}$ are of the form

$$
\begin{aligned}
& \mathbf{P}=\left[\begin{array}{cc}
\mathbf{P}_{1} & \mathbf{P}_{2} \\
\mathbf{0} & \mathbf{0}
\end{array}\right], \\
& \mathbf{Q}=\left[\begin{array}{ll}
\mathbf{Q}_{1} & \mathbf{Q}_{2} \\
\mathbf{Q}_{3} & \mathbf{Q}_{4}
\end{array}\right],
\end{aligned}
$$

where $\mathbf{P}_{1}$ and $\mathbf{Q}_{1}$ are square matrices of order $n_{1}, \mathbf{P}_{2}$ and $\mathbf{Q}_{2}$ are matrices of order $n_{1} \times n-n_{1}$. Moreover, $\mathbf{Q}_{4}$ and the corresponding null matrix in $\mathbf{P}$ are square matrices of order $n-n_{1}$.

Let us consider the case when $\mathbf{Q}_{4}$ is a singular matrix. As a result, we construct the matrix $\mathbf{R}=$ $\left[\begin{array}{ll}\mathbf{Q}_{3} & \mathbf{Q}_{4}\end{array}\right]$ of order $n_{1} \times n$. Using row operations find the rank of $\mathbf{R}$. Let the rank be $r$ and represent its submatrix structure as

$$
\mathbf{R}=\left[\begin{array}{cc}
\mathbf{0} & \mathbf{0} \\
\mathbf{Q}_{3}^{\prime} & \mathbf{Q}_{4}^{\prime}
\end{array}\right],
$$

where $\mathbf{Q}_{4}^{\prime}$ is a non-singular square matrix of order $r$. In the case $n_{1}>0, r=0, \mathbf{P} \lambda+\mathbf{Q}$ is a singular matrix. The rest of the algorithm analysis involves reorganizing the submatrices $\mathbf{P}$ and $\mathbf{Q}$, such that $\mathbf{P}_{1}$ and $\mathbf{Q}_{1}$ are square matrices of order $n-r$. The resulting system is similar to $\mathbf{P}$ and $\mathbf{Q}$ is (4), such that $\mathbf{Q}_{4}$ (which has order $r$ after these row operations and readjustment of elements in the submatrices) is nonsingular. 
Given $\mathbf{P}$ and $\mathbf{Q}$ corresponding to (4), the determinant of $P \lambda+Q$ is equal to determinant of

$$
\mathbf{G}(\lambda)=\left[\begin{array}{cc}
\mathbf{P}_{1} \lambda+\mathbf{Q}_{1}-\left(\mathbf{P}_{2} \lambda+\mathbf{Q}_{2}\right) \mathbf{Q}_{4}^{-1} \mathbf{Q}_{3} & 0 \\
\mathbf{Q}_{3} & \mathbf{Q}_{4}
\end{array}\right]
$$

Let

$$
\mathbf{G}_{1} \lambda+\mathbf{G}_{2}=\mathbf{P}_{1} \lambda+\mathbf{Q}_{1}-\left(\mathbf{P}_{2} \lambda+\mathbf{Q}_{2}\right) \mathbf{Q}_{4}^{-1} \mathbf{Q}_{3} .
$$

It follows that

$$
\operatorname{det} .(\mathbf{G}(\lambda))=\operatorname{det} .\left(\mathbf{G}_{1} \lambda+\mathbf{G}_{2}\right) \times \operatorname{det} .\left(\mathbf{Q}_{4}\right) .
$$

In case, $\mathbf{G}_{1}$ is non-singular, the problem can be reduced to an equivalent companion matrix, otherwise the above algorithm for computing a companion matrix of a system of the form $\mathbf{P} \lambda+\mathbf{Q}$ can be used recursively by substituting $\mathbf{G}_{1}$ and $\mathbf{G}_{2}$ for $\mathbf{P}$ and $\mathbf{Q}$, respectively. The fact that $\mathbf{G}_{1}$ and $\mathbf{G}_{2}$ are matrices of order less than $n$ implies that the algorithm terminates after a finite number of steps.

The complexity of this step can be as high as $O\left(n^{4}\right)$ in the worst case. However, such instance are very rare in practice and for most cases its complexity is bounded by $O\left(n^{3}\right)$. We will use this bound in the rest of the analysis.

For most practical cases the complexity of the univariate determinant algorithm is dominated by the reduction to upper Hessenberg matrix followed by the transformation to a similar Frobenius canonical form. As a matter of fact, both of these steps can be performed in $O\left(d^{3}+n^{3}\right)$ finite field operations. Furthermore the leading constant is small and is bounded by two for most cases. For most instances of the problem we expect that the Frobenius canonical form consists of one or two Frobenius matrices and as a result the polynomial multiplication is performed only a constant number of times. We present an $O\left(d^{3}+n^{3}\right)$ algorithm for reducing any matrix to upper Hessenberg form by similarity transformations. Given an upper Hessenberg matrix, $O\left(d^{3}+n^{3}\right)$ algorithm for reducing it to Frobenius canonical form is given in [Wil65].

The algorithm for reduction to upper Hessenberg form proceeds iteratively on the columns of the matrix. This algorithm is a simplified version of ELMHES in EISPACK library [GBDM77]. Let $c_{i, j}$ denote the element in the $i t h$ row and $j t h$ column of C. At the $k t h$ step it is assumed that $c_{k+1, k} \neq 0$. Otherwise we perform row and column interchange corresponding to similarity transformation by a permutation matrix. Let

$$
\mathbf{b}_{k+1}=\left[0, \ldots, 0,1,-\frac{c_{k+2, k}}{c_{k+1, k}}, \ldots,-\frac{c_{n, k}}{c_{k+1, k}}\right]^{T}
$$

represent an $n \times 1$ column vector whose first $k$ elements are zero. Let $\mathbf{B}_{k}$ represent an $n \times n$ matrix equivalent to the identity matrix for all but the $k+1^{s t}$ column. The $k+1^{s t}$ column of $\mathbf{B}_{k}$ is equal to $\mathbf{b}_{k+1}$. It is easy to see that $\mathbf{B}_{k}^{-1}$ is equivalent to $\mathbf{B}_{k}$, except that the $k+1^{s t}$ column of $\mathbf{B}_{k}^{-1}$ is $-\mathbf{b}_{k+1}$.

For each iteration of the algorithm for $k$ ranging from 1 to $d-1$ we compute $\mathbf{B}_{k}$ and $\mathbf{B}_{k}^{-1}$ and perform the similarity transformation

$$
\mathbf{C}=\mathbf{B}_{k} \mathbf{C B}_{k}^{-1} .
$$

At the end of the last iteration $\mathbf{C}=\mathbf{H}$, an upper Hessenberg matrix. The matrix multiplication at each step corresponds to multiplying by a column and row vector. As a result, there are $O\left((d+1-k)^{2}\right)$ finite field operations performed at the $k t h$ iteration. The running time of the overall transformation to upper Hessenberg matrix is $O\left(d^{3}\right)$. Moreover, the leading constant is bounded by $\frac{4}{3}$. A series of similarity transformations reduce the upper Hessenberg matrix to its Frobenius canonical form [Wil65]. It involves about $\frac{2}{3} d^{3}$ finite field operations.

\section{Multivariate Interpolation}

In this section we extend the algorithm for expanding univariate symbolic determinants to multivariate determinants. The algorithm uses multivariate interpolation and modular techniques as opposed to symbolic methods for computing determinants available in the computer algebra systems [MC91a].

Let us assume that each entry of the $n \times n$ matrix, $\mathbf{M}$, is a polynomial in $y_{1}, y_{2}, \ldots, y_{m}$. The determinant is a polynomial of the form $F\left(y_{1}, \ldots, y_{m}\right)$. For most resultant formulations the entries are linear polynomials. Furthermore, we assume that we have a tight bound on the degree of the polynomial. Let the maximum degree of $y_{i}$ in $F\left(y_{1}, y_{2}, \ldots, y_{m}\right)$ be $d_{i}$. This degree bound can always be computed by adding the degrees of $y_{i}$ in the rows or columns of $M$. As a result, $F$ can have at most $q_{1}=\left(d_{1}+1\right)\left(d_{2}+1\right) \ldots\left(d_{m}+1\right)$ terms. In some applications it is simpler to compute the total degree of $F\left(y_{1}, \ldots, y_{m}\right)$. Let that degree be $d$ and the resulting polynomial can have atmost $q_{1}=C(m, d)$ coefficients, where

$$
C(m, d)=\left(\begin{array}{c}
m+d-1 \\
d
\end{array}\right) .
$$

\subsection{Dense Interpolation}

In this section we consider the dense multivariate in terpolation algorithm for determinant computation 
and improve it using the univariate determinant algorithm presented in the previous section.

We express $F$ as a polynomial of the

$$
F\left(y_{1}, \ldots, y_{m}\right)=c_{1} m_{1}+c_{2} m_{2}+\ldots+c_{q} m_{q}
$$

where $q$ corresponds to $q_{1}$ or $q_{2}$. The $m_{i}=$ $y_{1}^{d_{1, z}} y_{2}^{d_{2,3}} \ldots y_{m}^{d_{m, 2}}$ are distinct monomials and $c_{i}$ are the corresponding coefficients. The problem of determinant computation reduces to enumerating the $m_{i}$ 's and solving for $c_{i}$ 's. The latter step is performed by using Vandermonde interpolation in [MC91a]. More details on Vandermonde interpolation are given in [Zip90]. In particular, choose $m$ distinct primes, $p_{1}, \ldots, p_{m}$ and let $b_{i}=p_{1}^{d_{1,2}} p_{2}^{d_{2,2}} \ldots p_{m}^{d_{m, 2}}$. Distinct monomials, $m_{i}$ and $m_{j}$, evaluate to different values. Let $a_{s}=F\left(p_{1}^{i}, p_{2}^{i}, \ldots, p_{m}^{i}\right), i=1, q$, be the value of the function computed using Gauss elimination. The resulting problem is reduced to solving a $q \times q$ Vandermonde system. The overall running time is $O\left(q n^{3}+q^{2}\right)$. This method is useful for lower values of $m$. Consider the case when $d_{i}=n$ and $q=q_{2}=(n+1)^{m}$. As a result the overall running time is $O\left(n^{m+3}+n^{2 m}\right)$. In practice the function evaluations account for more than half of the running time. In the case of Macaulay's formulation $d_{i}<n / m$ and as a result more time is spent in function evaluation as compared to Vandermonde interpolation.

Without loss of generality we assume that $d_{m}=$ $\max \left(d_{1}, d_{2}, \ldots, d_{m}\right)$. Express $\mathbf{M}$ as a matrix polynomial in $y_{m}$ as

$$
\mathbf{M}=\mathbf{M}_{\mathbf{d}_{\mathbf{m}}} y_{m}^{d_{m}}+\ldots+\mathbf{M}_{\mathbf{1}} y_{m}+\mathbf{M}_{\mathbf{0}}
$$

where $\mathbf{M}_{\mathbf{d}_{\mathbf{m}}}, \ldots, \mathbf{M}_{\mathbf{1}}, \mathbf{M}_{\mathbf{0}}$ are matrices whose elements are polynomial functions in $y_{1}, \ldots, y_{m-1}$. The rest of the algorithm proceeds iteratively. At the $i t h$ iteration:

1. Substitute $\left(y_{1}, \ldots, y_{m-1}\right)=\left(p_{1}^{i}, \ldots, p_{m-1}^{i}\right)$ in $\mathbf{M}$ and treat it as a univariate matrix polynomial in $y_{m}$.

2. Compute the determinant of the matrix polynomial using the algorithm highlighted in the previous section. The resulting polynomial cor-responds to $F\left(p_{1}^{i}, p_{2}^{i}, \ldots, p_{m-1}^{i}, y_{m b}\right)$.

Let us express $F$ as a polynomial in $y_{m}$. That is:

$$
F\left(y_{1}, y_{2}, \ldots, y_{m}\right)=\Sigma_{i=0}^{n} F_{i}\left(y_{1}, \ldots, y_{m-1}\right) y_{m}^{i} \text {. }
$$

At the end of $i^{\text {th }}$ iteration we know the values of

$$
F_{0}\left(p_{1}^{i}, p_{2}^{i}, \ldots, p_{m-1}^{i}\right), \ldots, F_{d_{m}}\left(p_{1}^{i}, p_{2}^{\dot{i}}, \ldots, p_{m-1}^{i}\right)
$$

This steps are repeated for $i=1, q$, where $q=$ $\left(d_{1}+1\right) \ldots\left(d_{m-1}+1\right)$. Each of the $F_{i}$ 's is computed using Vandermonde interpolation and has upto $q$ terms. The running time of the resulting algorithm is $O\left(q p^{3}+d_{m} q^{2}\right)$, where $p=\max \left(n, d_{m}\right)$. In the event $d_{i}=n, q=(n+1)^{m-1}$ and the overall running time is $O\left(n^{m+2}+n^{2 m-1}\right)$.

In Macaulay's, Sylvester's and some of Dixon's formulation of resultants, each entry of the matrix is a linear polynomial in the coefficients of the given system of equations. As a result we express $\mathbf{M}$ as

$$
\mathbf{M}=\mathbf{M}_{\mathbf{1}} y_{m}+\overline{\mathbf{M}},
$$

where $\mathbf{M}_{\mathbf{1}}$ is a numeric matrix and entries of $\overline{\mathbf{M}}$ are polynomials in $y_{1}, \ldots, y_{m-1}$. We either multiply $\overline{\mathbf{M}}$ by $\mathbf{M}_{\mathbf{1}}^{-1}$ or use the transformations presented in the previous section to reduce it to characteristic polynomial computation problem. Since computing the characteristic polynomial involves a constant factor as compared to determinant, we have reduced the symbolic complexity by one variable.

\subsection{Sparse Interpolation}

The dense interpolation algorithm is practical for small values of $m$ (upto three or four). This is due to the fact that $q$ is of the order of $d_{\dot{i}}^{m}$. Furthermore, the determinant, $F\left(y_{1}, \ldots, y_{m}\right)$, may be a sparse polynomial. To circumvent these problems Manocha and Canny considered sparse interpolation algorithms for determinant computation [MC91b]. These include deterministic and probablistic algorithms. The deterministic versions of Ben-Or and Tiwari, [BoT88], and Zippel, [Zip90], require an upper bound on the number of terms in the resulting polynomial, which is difficult for symbolic determinants. On the other hand, Zippel's probablistic algorithm, [Zip90], works well for our applications. Techniques to improve its probability of success using modular methods are presented in [MC91b]. In this section we use the univariate determinant algorithm to improve its performance.

\subsubsection{Zippel's Algorithm}

Zippel's probablistic algorithm proceeds inductively. It only expects a bound on the degree of each variable in the resulting polynomial, $d_{i}$. Furthermore, its performance is a function of the number of terms in the output. We present a brief outline here and more details are given in [Zip90].

Choose $m$ random numbers $r_{1}, \ldots, r_{m}$ from the coefficient field used for defining the polynomial coefficients. The algorithm introduces a variable at each 
stage. At the $k+1$ th stage it has interpolated a polynomial in $k$ variables and is of the form:

$$
\begin{gathered}
F_{k}\left(y_{1}, \ldots, y_{k}\right)=F\left(y_{1}, y_{2}, \ldots, y_{k}, r_{k+1}, \ldots, r_{m}\right) \\
=c_{1, k} m_{1, k}+\ldots+c_{\alpha_{k}, k} m_{\alpha_{k}, k}
\end{gathered}
$$

where $m_{i, k}, l=1, \alpha_{k}$, represents a monomial in $y_{1}, y_{2}, \ldots, y_{k}$. As a result, $F_{k}$ is a polynomial in $k$ variables having $\alpha_{k}$ terms, where $\alpha_{k} \leq q$. To compute $F_{k+1}\left(y_{1}, \ldots, y_{k+1}\right)$ from $F_{k}\left(y_{1}, \ldots, y_{k}\right)$, Zippel's algorithm represents $F_{k+1}$ as

$$
\begin{gathered}
F_{k+1}\left(y_{1}, \ldots, y_{k+1}\right)=F\left(y_{1}, \ldots, y_{k+1}, r_{k+2}, \ldots, r_{n}\right) \\
=h_{1}\left(y_{k+1}\right) m_{1, k}+\ldots+h_{\alpha_{k}}\left(y_{k+1}\right) m_{\alpha_{k}, k},
\end{gathered}
$$

where each each $h_{i}\left(y_{k+1}\right)$ is a polynomial of degree $d_{k+1}$. Let us represent $h_{i}\left(y_{k+1}\right)$ as

$$
h_{i}\left(y_{k+1}\right)=h_{i, 0}+h_{i, 1} y_{k+1}+\ldots+h_{i, d_{k+1}} y_{k+1}^{d_{k+1}} .
$$

Each $h_{i}\left(y_{k+1}\right)$ is computed using Vandermonde interpolation. In other words, the algorithm requires to know the value of $h_{i}\left(p_{k+1}^{j}\right)$ for $1 \leq i \leq \alpha_{k}, 0 \leq$ $j \leq d_{k+1}$. The algorithm proceeds iteratively on $i$ and $j$ in the following manner:

- For $0 \leq j \leq d_{k+1}$ do:

- For $1 \leq i \leq \alpha_{k}$ do: Compute

$$
F\left(p_{1}^{i}, \ldots, p_{k}^{i}, p_{k+1}^{j}, r_{k+2}, \ldots, r_{m}\right) .
$$

It corresponds to the value of the polynomial

$$
h_{1}\left(p_{k+1}^{j}\right) b_{1, k, i}+\ldots+h_{\alpha_{k}}\left(p_{k+1}^{j}\right) b_{\alpha_{k}, k, i},
$$

where

$$
b_{l, k, i}=m_{l, k}{ }_{\left(y_{1}=p_{1}^{2}, y_{2}=p_{2}^{z}, \ldots, y_{k}=p_{k}^{i}\right)} .
$$

- Solve the resulting $\alpha_{k} \times \alpha_{k}$ Vandermonde system $h_{1}\left(p_{k+1}^{j}\right), h_{2}\left(p_{k+1}^{j}\right), \ldots, h_{\alpha_{k}}\left(p_{k+1}^{j}\right)$.

- Compute $h_{i}\left(y_{k+1}\right)$ by solving for a $\left(d_{k+1}+1\right) \times$ $\left(d_{k+1}+1\right)$ Vandermonde system. This is repeated for $1 \leq i \leq \alpha_{k}$.

- Substitute $h_{i}\left(y_{k+1}\right)$ to represent $F_{k+1}$ as a polynomial in $k+1$ variables. $F_{k+1}\left(y_{1}, \ldots, x_{y+1}\right)$ can have at most $\left(\alpha_{k} *\left(d_{k+1}+1\right)\right) \leq q$ terms.

The algorithm starts with $F\left(r_{1}, \ldots, r_{n}\right)$ and computes the $n$ stages. At the $k+1$ th stage the algorithm involves $\alpha_{k} *\left(d_{k+1}+1\right)$ function evaluations and solving $\left(d_{k+1}+1\right)$ Vandermonde systems of $\alpha_{k}$ unknowns and $\alpha_{k}$ Vandermonde systems with $d_{k+1}+1$ unknowns. Thus, the running time at the $k+1$ th stage is $O\left(\alpha_{k} d_{k+1} n^{3}+d_{k+1} \alpha_{k}^{2}+\alpha_{k} d_{k+1}^{2}\right)$. In the worst case, $\alpha_{k}$ is bounded by $q$.

\subsubsection{Improved Sparse Interpolation}

To improve the performance of Zippel's algorithm we use the univariate determinant algorithm. At the $k+1$ th stage our algorithm proceeds in the following manner:

- For $1 \leq i \leq \alpha_{k}$ do:

Compute $F\left(p_{1}^{i}, p_{2}^{i}, \ldots, p_{k}^{i}, y_{k+1}, r_{k+2}, \ldots, r_{m}\right)$ using the univariate determinant algorithm. It corresponds to a polynomial of the form

$$
h_{1}\left(y_{k+1}\right) b_{1, k, i}+\ldots+h_{\alpha_{k}}\left(y_{k+1}\right) b_{\alpha_{k}, k, i} .
$$

- For $0 \leq j \leq d_{k+1}$ do:

- Compute $h_{1, j}, h_{2, j} \ldots, h \alpha_{k}, j$ by solving the $\alpha_{k} \times \alpha_{k}$ Vandermonde system.

- Substitute $h_{i}\left(y_{k+1}\right)$ 's to compute $F_{k+1}$.

The running time of the $k+1$ th iteration of the improved algorithm is $O\left(\alpha_{k} p^{3}+d_{k+1} \alpha_{k}^{2}\right)$, where $p=$ $\max \left(n, d_{k+1}\right)$. In general, $d_{k+1}$ is bounded by $n$ as each entry of the matrix is a linear polynomial in the unknowns.

\section{Implementation}

The improved algorithms for resultant computation have been implemented as part of our package for resultant computation [MC91b]. The package has been developed in $\mathrm{C}++$ on Sun-4's and the code has been ported on an IBM RS/6000 for performance analysis. Many routines for linear algebra are available from standard libraries. However, they are mostly implemented in Fortran and used with floating point arithmetic as opposed to exact or modular integer arithmetic. One of the nice features of these algorithms is their storage requirement. The total amount of space is a linear function of the input and output size. That includes the symbolic matrices and the multivariate polynomials. The space requirement for Vandermonde interpolation is linear in the size of the symbolic matrices corresponding to resultant formulation and the output polynomial.

In this section we compare the performance of different elimination algorithms for implicitizing parametric surfaces. More experiments are planned for other applications. These include comparisons with implementations of Gröbner basis in Cocoa and Macaulay computer algebra systems. 


\begin{tabular}{|c|c|c|c|}
\hline Algorithm & Time & Machine & Reference \\
\hline \hline Gröbner bases & $10^{5}$ sec. & Symbolics 3650 & Hoffmann [Hof90] \\
\hline Ritt-Wu's algorithm & $28,000 \mathrm{sec}$ & Sun-3 & Gao and Chou [GC90] \\
\hline Multipolynomial resultants & $138 \mathrm{sec}$. & IBM RS/6000 & Manocha and Canny [MC91b] \\
\hline Improved multipolynomial resultants & $24 \mathrm{sec}$. & IBM RS/6000 & Section 3 \\
\hline \hline
\end{tabular}

Table 1: The performance of different implicitization algorithms on (6)

\subsection{Implicitization}

We applied the results of this algorithm to implicitizing rational parametric surfaces. Implicitization is a fundamental problem in geometric and solid modeling and also seems to be a standard benchmark for elimination algorithms. Given a parametrization, expressed in projective coordinates,

$$
(x, y, z, w)=(X(s, t), Y(s, t), Z(s, t), W(s, t)),
$$

we formulate the parametric equations

$$
\begin{aligned}
& w X(s, t)-x W(s, t)=0 \\
& w Y(s, t)-y W(s, t)=0 \\
& w Z(s, t)-z W(s, t)=0
\end{aligned}
$$

and the problem of implicitization corresponds to computing the resultant of these equations by considering them as polynomials in $s$ and $t$ [MC92]. Other algorithms for implicitization include Gröbner bases and Ritt-Wu's algorithm. Hoffmann has surveyed these techniques in [Hof92]. A particular benchmark for implicitization has been a bicubic parametric surface given by Hoffmann, [Hof90]:

$$
\begin{aligned}
x= & -3 t(t-1)^{2}+(s-1)^{3}+3 s \\
y= & 3 s(s-1)^{2}+t^{3}+3 t \\
z= & -3\left(s\left(s^{2}-5 s+5\right) t^{3}+\left(s^{3}+6 s^{2}-9 s+1\right) t^{2}\right. \\
& \left.-\left(2 s^{3}+3 s^{2}-6 s+1\right) t+s(s-1)\right)
\end{aligned}
$$

According to resultant algorithms, the problem of implicitization reduces to expanding a symbolic determinant whose order is equal to the degree of the implicit equation. Furthermore, each entry of the matrix is a linear homogeneous polynomial in $x, y, z, w$. This is equivalent to a non-homogeneous polynomial in $x, y, z$. We used the improved dense multivariate interpolation algorithm, presented in Section 3, for this example. The performance of different algorithms is given in Table 1.

Thus, we see that the multipolynomial resultants give us the best performance for implicitization.

This is after taking into account the relative performance of different machines. Furthermore, the improved dense interpolation algorithm gives us a speed-up of 5.5 as compared to the previous algorithm presented in [MC91a] on this benchmark. In this case, the implicit equation is a polynomial of degree 18. The relative performance improves for parametrizations with higher implicit degrees.

\subsection{Implicitizing Parametrizations with Base Points}

A base point of a parametrization is the common root of $X(s, t), Y(s, t), Z(s, t), W(s, t)$. They also include the points at infinity. Direct applications of resultants or Gröbner bases fail to implicitize such parametrizations. Modified algorithms using resultants and Gröbner basis are presented by Manocha and Canny, [MC92] and Kalkbrener, [Kal91], respectively. Again the resultant based algorithms perform better. In particular, the algorithm in [MC92] considers a perturbed system of the form:

$$
\begin{gathered}
w X(s, t)-x W(s, t)=0 \\
w Y(s, t)-y W(s, t)=0 \\
w Z(s, t)-z W(s, t)+\lambda G(s, t)=0,
\end{gathered}
$$

where $G(s, t)$ is a random polynomial. The resultant is a polynomial of the form $R(x, y, z, \lambda)$. Let us express the resultant as a polynomial in $\lambda$ and say the coefficient of the lowest degree term is $P(x, y, z)$. We are only interested in $P(x, y, z)$ as opposed to the entire polynomial $R(x, y, z, \lambda) . P(x, y, z)$ can be decomposed as $P(x, y, z)=F(x, y, z) G(x, y)$, where $F(x, y, z)$ is the implicit equation and $G(x, y)$ corresponds to the projection of seam curves (images of base points) [MC92]. Given a prime $p$, the computation of $P(x, y, z) \bmod p$ using dense interpolation algorithm takes about $1180 \mathrm{sec}$. on an IBM RS/6000 for a bicubic parametrization with base points [MC92]. Using the improved interpolation algorithms presented in Section 3, computation of $P(x, y, z) \bmod p$ takes about $58 \mathrm{sec}$. on IBM RS/6000 for the same parametrization. Thus, we see a speed-up of 20.5 by using algorithms presented in this paper over the 
earlier algorithm based on resultants and multivariate interpolation.

\section{Acknowledgements}

We are grateful to James Demmel for productive discussions. This research was supported in part by David and Lucile Packard Fellowship and National Science Foundation Presidential Young Investigator Award (\# IRI-8958577). The first author is also supported by IBM Graduate Fellowship.

\section{References}

[BoT88] M. Ben-or and P. Tiwari. A deterministic algorithm for sparse multivariate polynomial interpolation. In $A C M S y m$ posium on Theory of Computing, pages 301-309, 1988.

[Buc85] B. Buchberger. Groebner bases: An algorithmic method in ideal theory. In N.K. Bose, editor, Multidimensional Systems Theory, pages 184-232. D. Reidel Publishing Co., 1985.

[Buc89] B. Buchberger. Applications of groebner bases in non-linear computational geometry. In D. Kapur and J. Mundy, editors, Geometric Reasoning, pages 415 447. MIT Press, 1989.

[Can88] J.F. Canny. The Complexity of Robot Motion Planning. ACM Doctoral Dissertation Award. MIT Press, 1988.

[Can90] J.F. Canny. Generalized characteristic polynomials. Journal of Symbolic Computation, 9:241-250, 1990.

[Dix08] A.L. Dixon. The eliminant of three quantics in two independent variables. Proceedings of London Mathematical Society, 6:49-69, 209-236, 1908.

[GBDM77] B.S. Garbow, J.M. Boyle, J. Dongarra, and C.B. Moler. Matrix Eigensystem Routines - EISPACK Guide Extension, volume 51 of Lecture Notes in Computer Science. Springer-Verlag, Berlin, 1977.

[GC90] X.S. Gao and S.C. Chou. Implicitization of rational parametric equations. Technical report, Department of Computer Science TR-90-34, University of Texas at Austin, 1990.

[GLR82] I. Gohberg, P. Lancaster, and L. Rodman. Matrix Polynomials. Academic Press, New York, 1982.
[Hof90] C.M. Hoffmann. Algebraic and numeric techniques for offsets and blends. In W. Dahmen, M. Gasca, and C. Micchelli, editors, Computations of Curves and Surfaces, pages 499-528. Kluwer Academic Publishers, 1990.

[Hof92] C. Hoffmann. Implicit curves and surfaces in computer aided geometric design. Technical report, Department of Computer Science CER-92-002, Purdue University, 1992.

[Jou89] Jean-Pierre Jouanolou. Le formalisme du résultant. Technical report, Université Louis Pasteur, Department of Mathematics, France, 1989. Manuscript.

[Kal91] M. Kalkbrener. Three Contributions to Elimination Theory. PhD thesis, Technisch-Naturwissenschaftliche Fakultat, Johannes Kepler Universitat, Linz, 1991.

[KL88] E. Kaltofen and Y.N. Lakshman. Improved sparse multivariate polynomial interpolation algortihms. In Lecture Notes in Computer Scrence, volume 358, pages 467-474. Springer-Verlag, 1988.

[KL92] D. Kapur and Y.N. Laksman. Elimination methods: An introduction. In D. Kapur B. Donald and J. Mundy, editors, Symbolic and Numerical Computation: an Integration. Academic Press, 1992. To Appear.

[Mac02] F.S. Macaulay. On some formula in elimination. Proceedings of London Mathematical Society, pages 3-27, May 1902.

[Mac21] F.S. Macaulay. Note on the resultant of a number of polynomials of the same degree. Proceedings of London Mathematical Society, pages 14-21, June 1921.

[Mac64] F.S. Macaulay. The Algebratc Theory of Modular Systems. Stechert-Hafner Service Agency, New York, 1964.

[MC27] F. Morley and A.B. Coble. New results in elimination. American Journal of Mathematics, 49:463-488, 1927.

[MC91a] D. Manocha and J.F. Canny. Efficient techniques for multipolynomial resultant algorithms. In Proceedings of Internatronal Symposium on Symbolic and Algebraic Computation, pages 86-95, 1991.

[MC91b] D. Manocha and J.F. Canny. Multipolynomial resultant algorithms. In Proceedings of International Symposium on Intelligent Robotics, pages 348-358, 
1991. Also available as Technical Report UCB/CSD 91/632, Computer Science Division, University of California, Berkeley.

[MC92] D. Manocha and J.F. Canny. The implicit representation of rational parametric surfaces. Journal of Symbolic Computation, 1992. To appear.

[Rit50] J.F. Ritt. Differential Algebra. AMS Colloquium Publications, 1950.

[Sal85] G. Salmon. Lessons Introductory to the Modern Higher Algebra. G.E. Stechert \& Co., New York, 1885.

[Stu91] B. Sturmfels. Sparse elimination theory. Technical Report 91-32, Mathematical Sciences Institute, Cornell University, 1991.

[SZ91] B. Sturmfels and A. Zelevinsky. Multigraded resultants of sylvester type. Technical report, Mathematical Sciences Institute, Cornell University, 1991. Manuscript.

[Wae50] B.L. Van Der Waerden. Modern Algebra (third edution). F. Ungar Publishing Co., New York, 1950.

[Wil65] J.H. Wilkinson. The algebraic eigenvalue problem. Oxford University Press, Oxford, 1965.

[Wu78] W. Wu. On the decision problem and the mechanization of theorem proving in elementary geometry. Scientia Sinica, 21:150-172, 1978. Also in Bledsoe and Loveland, eds. Theorem Proving: After 25 years, Contemporary Mathematics, 29, 213-234.

[Zip90] R. Zippel. Interpolating polynomials from their values. Journal of Symbolic Computation, 9:375-403, 1990. 